\title{
Cartas percentílicas da proficiência motora de crianças com idades entre os seis e os 10 anos
}

http://dx.doi.org/10.11606/1807-5509202000040639

\author{
Luciana Ferreira $^{* / * *}$ \\ J osé Luiz Lopes Vieira* \\ Pâmela Norraila da Silva* \\ Franciele Ferreira da Rocha* \\ Priscila Caçola** \\ Raquel Nichele de Chaves***
} *Universidade Estadual
de Maringá, Maringá,
PR, Brasil.
**University of Texas
at Arlington, Arlington,
TX, EUA.
*** Uni ve r sida de
Tecnológica Federal
do Paraná, Curitiba,
PR, Brasil.

\section{Resumo}

Este estudo teve como objetivos principais (i) apresentar valores normativos para os oito subtestes do Teste de Proficiência Motora Bruininks-Oseretsky (BOT-2) e (ii) contrastar com os valores sugeridos no manual do teste original. A amostra foi constituída por 931 crianças com idade entre os seis e 10 anos, de ambos os sexos, provenientes do município de Maringá-PR. Para a avaliação da proficiência motora foi utilizado o BOT-2. A construção dos valores de referência percentílica foi efetuada com base no método LMS, implementado no software LMSchartmaker Pro versão 2.54. As meninas obtiveram maiores escores nos subtestes precisão motora fina, integração motora fina, destreza manual e equilibrio. Os meninos apresentaram maiores escores na coordenação de membros superiores, velocidade e agilidade e força muscular. As crianças maringaenses avaliadas apresentaram valores superiores às crianças norte-americanas nos subtestes de coordenação bilateral, velocidade e agilidade, equilíbrio e coordenação dos membros superiores. Os resultados expressam a forte variabilidade interindividual em termos de proficiência motora, bem como diferenças entre meninos e meninas. As curvas ilustram o aumento dos níveis de proficiência motora ao longo das idades, com trajetórias distintas para cada subteste. Desse modo, a utilização dos valores normativos apresentados neste estudo poderá auxiliar a ação pedagógica nos contextos escolares e esportivos, contribuindo para o planejamento de intervenções motoras mais efetivas.

Palavras-chave: Proficiência Motora; Bruininks-Oseretsky; Valores de referência.

\section{Introdução}

A capacidade de realizar várias habilidades motoras como correr, chutar, saltar de forma proficiente é muitas vezes definida como proficiência motora ${ }^{1,2}$. Baixos níveis de proficiência motora podem influenciar negativamente crianças e jovens, reduzir sua motivação para a prática de atividade física, conduzi-las a um comportamento cada vez mais sedentário ${ }^{3}$ com fortes prejuízos à saúde .

A baixa proficiência motora tem sido associada ao menor prazer na realização das tarefas diárias ${ }^{5}$, ansiedade e depressão $0^{6}$, habilidades sociais precárias e baixa autoestima ${ }^{7}$, diminuição da qualidade de vida ${ }^{8}$, menor aptidão cardiorrespiratória ${ }^{9}$ e obesidade ${ }^{10,11}$. Na ausência de intervenção, muitas dessas dificuldades podem persistir e continuar interferindo no desempenho de tarefas cotidianas, além de conduzir a um engajamento pobre em atividades físicas na vida adulta ${ }^{2,6,12}$. Dada a influência negativa da baixa proficiência motora na qualidade de vida desde a infância, sua identificaçáo precoce, por meio de uma avaliaçẫo efetiva, é crucial; consequentemente, as informações obtidas podem ser uma ferramenta importante para programas de intervenção ${ }^{13}$.

Mediante a importância da avaliação da proficiência motora na infância, diferentes baterias de testes padronizados foram desenvolvidas e disponibilizadas 
na literatura, entre as quais, destacam-se o TGMD ${ }^{14}$ (Test of Gross Motor Development), MABC ${ }^{15}$ (Movement Assessment Battery for Children), KTK ${ }^{16}$ (Körperkoordinationstest Für Kinder) e o BOT-2 ${ }^{17}$ (Bruininks-Oseretsky Test of Motor Proficiency, Second Edition). Nesse âmbito, o BOT-2 apresenta uma grande abrangência de componentes e fornece uma visão global sobre o nível de proficiência motora da criança ${ }^{17}$. Entre as principais qualidades, o BOT-2 possui de boa a excelente confiabilidade dos dados ${ }^{17,18}$, categoriza a proficiência motora para uma ampla faixa etária ( $4 \mathrm{a}$ 21 anos) $)^{19}$, tem uma avaliação motora detalhada com subtestes que podem ser avaliados separadamente ${ }^{18}$, e avalia algumas das tarefas que correspondem aos contextos de vida mais amplos referidos no Critério $\mathrm{B}$ do DSM-V ${ }^{20}$. Entre os fatores que podem limitar seu uso, ressalta-se o custo para compra do equipamento e o tempo de administraçáo ${ }^{21}$.

A construçáo do BOT-2 foi realizada com base em uma amostra de 1520 participantes, com idades compreendidas entre os quatro a 21 anos, provenientes de diferentes locais dos Estados Unidos ${ }^{17}$. Inicialmente, o BOT-2 era utilizado para identificar as crianças com problemas motores. Atualmente é considerado uma das baterias mais utilizadas internacionalmente ${ }^{22,23}$. Desse modo, uma cuidadosa avaliação é requerida, especialmente no que concerne às normas e aos pontos de corte sugeridos para outras populaçóes. Níveis de proficiência motora estão condicionados a um grande conjunto de fatores biológicos e ambientais ${ }^{24,25}$ e, em termos populacionais, podem apresentar forte variabilidade.

Estudos prévios têm sugerido a construção de valores percentílicos para avaliar e monitorizar a coordenação motora e neuromotora de crianças e jovens. Por exemplo, desempenho da coordenação motora grossa

\section{Método}

Este estudo está integrado ao projeto institucional submetido e aprovado pelo Comitê Permanente de Ética em Pesquisa com Seres Humanos da Faculdade Assis Gurgacz, sob parecer no. 1.207.141/2015.

\section{Participantes}

A população foi composta por estudantes de seis a 10 anos de idade, de ambos os sexos, matriculados em escolas públicas e privadas do ensino Fundamental, do município de Maringá-PR. A cidade de Maringá, com base na bateria de testes KTK em amostras portuguesas $^{26,27}$, belga $^{28}$ e peruana ${ }^{29}$; e coordenaçấo neuromotora a partir da bateria Zurich Neuromotor Assessment em uma amostra suíça ${ }^{30}$. Tais pesquisas sugerem valores normativos para as populaçóes específicas avaliadas. No Brasil, SiLva et al. ${ }^{31}$ propóe valores percentílicos para o desempenho motor de crianças da regiāo do Cariri, no Ceará, com a avaliação de componentes motores como força muscular, aptidão cardiorrespiratória, agilidade, velocidade e flexibilidade.

A lacuna na literatura sobre valores normativos de crianças brasileiras referente à proficiência motora pode ser justificada pela grande extensão geográfica, diversidade cultural, étnica e socioeconômica, dificultando a aplicação e padronização de testes motores, bem como a construçáo de valores de referência ${ }^{31}$. Por outro lado, diferenças ambientais, culturais e até mesmo genéticas podem ser observadas dentro de um mesmo país ou região, relacionadas sobretudo às condiçôes sociais, de saúde e das próprias rotinas diárias das crianças e, assim, exigir leituras mais precisas e mais próximas da realidade ${ }^{32}$.

As cartas percentílicas possibilitam aos professores de educação física e terapeutas valores de referência da proficiência motora que podem ser utilizados para propor e implementar propostas didáticometodológicas adequadas ao perfil dos alunos. Especificamente, pode auxiliar nas práticas avaliativas descritivas, que compóem o currículo dos anos iniciais do ensino fundamental, permitindo a análise e interpretaçáo do processo de ensino/aprendizagem da criança. Com base nas consideraçóes anteriores, os propósitos do presente estudo são (i) apresentar valores de referência percentílica para oito subtestes do BOT-2; (ii) e contrastar os resultados com os valores determinados no manual do teste original.

especificamente, situada na região Norte Central Paranaense, tem aproximadamente 403.063 habitantes e apresenta um Índice de Desenvolvimento Humano (IDH) de 0,808 , considerado elevado.

O cálculo amostral foi realizado com base nos dados da Secretaria de Educação de Maringá, de acordo com o número de crianças matriculadas nas 49 escolas públicas e no site do Instituto Nacional de Estudos e Pesquisas Educacionais Anísio Teixeira neste caso, para obter a quantidade de crianças matriculadas nas 34 escolas privadas. No ano de 2015, havia16.335 
crianças matriculadas no ensino fundamental da rede municipal de ensino e 7.362 no ensino privado. Após esse levantamento, foi realizado um cálculo amostral, seguindo a fórmula proposta por МоттА ${ }^{33}$ para estabelecer o número necessário de crianças de seis a 10 anos que representassem a cidade de Maringá. Sendo assim, 603 crianças matriculadas nas escolas públicas e 328 crianças matriculadas nas escolas privadas do município de Maringá foram incluídas na amostra, totalizando 931. A amostra foi estratificada por sexo e idade. Em cada valor discreto de idade foram incluídas as crianças cuja idade estivesse compreendida entre o valor inteiro e noventa e nove decimais. Por exemplo, uma criança seria considerada no grupo seis anos caso tivesse uma idade entre 6,0 e 6,99 anos decimais.

TABELA 1 - Distribuição amostral em função do sexo e idade.

\begin{tabular}{llcc}
\hline Idade & Meninos & Meninas & Total \\
\hline 6 & 45 & 50 & 95 \\
7 & 80 & 97 & 177 \\
8 & 128 & 120 & 248 \\
9 & 133 & 135 & 268 \\
10 & 68 & 75 & 143 \\
\hline Total & 454 & 477 & 931 \\
\hline
\end{tabular}

\section{Instrumentos}

Para avaliar a proficiência motora foi utilizado o Teste de Proficiência Motora de Bruininks-Oseretsky - BOT-2 ${ }^{17}$. O BOT-2 possui 53 tarefas, que podem ser agrupadas a oito subtestes. Para este estudo foi utilizado o Point Score Total dos oito subtestes (precisão motora fina, integração motora fina, destreza manual, coordenação de membros superiores, coordenação bilateral, equilíbrio, velocidade e agilidade e força). $\mathrm{O}$ QUADRO 1 descreve os subtestes, bem como a faixa de pontuação bruta, somada a partir das pontuaçóes de desempenho bruto nos 53 itens do BOT-2, o que permite que o desempenho nos itens de um participante seja mensurado numa escala gradual.

A soma das pontuaçóes resulta em uma proficiência motora total, que pode ser classificada em "bem acima da média" (percentil acima de 98), "acima da média” (percentil 84-97), "média" (percentil 18-83), "abaixo da média" (percentil 3-17), ou "bem abaixo da média" (percentil abaixo de 3), conforme o manual do BOT-2 ${ }^{17}$.

QUADRO 1 - Subtestes do BOT-2.

\begin{tabular}{|lc|}
\hline Subtestes & Pontuação (pontos) \\
\hline Precisão Motora Fina & $0-41$ \\
Integração Motora Fina & $0-40$ \\
Destreza Manual & $0-45$ \\
Coordenação dos Membros Superiores & $0-39$ \\
Coordenação Bilateral & $0-24$ \\
Equilíbrio & $0-37$ \\
Velocidade e Agilidade & $0-52$ \\
Força Muscular & $0-42$ \\
\hline
\end{tabular}




\section{Procedimentos}

Foram agendados os dias e horários para administrar a avaliação do BOT-2. Três pesquisadores treinados administraram a avaliaçáo em um espaço isolado na escola de cada criança. O local de realizaçáo do teste selecionado estava distante de ruídos, distraçóes e os materiais foram organizados previamente. Cada criança foi avaliada individualmente durante a execuçáo das 53 tarefas, cuja aplicação tinha duração de 35 a 60 minutos. Inicialmente, o avaliador fornecia para a criança as instruçôes necessárias para cada tarefa. A sequência dos testes seguiu rigorosamente a proposta do manual, iniciando pelas tarefas de destreza manual e finalizando com tarefas de força, pois inverter a ordem de aplicação poderia comprometer o desempenho motor em determinadas tarefas, devido ao cansaço e/ ou à fadiga. Quando necessário, foi solicitada a retirada de acessórios como bonés, presilhas, dentre outros, que pudessem inviabilizar a mensuração correta das tarefas.

\section{Análise de dados}

Após a análise exploratória dos dados para a verificação de possíveis erros de entrada e

\section{Resultados}

A TABELA 2 mostra os resultados descritivos de ambos os sexos nas diferentes idades para os oito subtestes do BOT-2. Em geral, meninos e meninas apresentam incrementos nos valores médios com o avanço da idade. Destaca-se os valores máximos alcançados em todas as idades pelas meninas nos subtestes de Equilíbrio (37 pontos) e Coordenaçáo Bilateral (24 pontos).

Na FIGURA 1, estão representadas as curvas de referência percentílicas para os oito subtestes do BOT2. Em todos os subtestes, observa-se aumento dos níveis de proficiência motora ao longo das idades, cuja a magnitude está condicionada ao sexo e ao subteste. Em geral, as meninas possuem escores mais elevados nos subtestes de precisão motora fina, integração motora fina, destreza manual e equilíbrio. Por sua vez, os meninos apresentam escores mais elevados nos subtestes de coordenação dos membros superiores, velocidade e agilidade e força muscular. No subteste de coordenação bilateral, as meninas apresentam escores superiores até os 8 anos. valores extremos, foram calculados valores médios, respectivos desvios-padrão e medidas de amplitude (mínimo e máximo). Os valores percentílicos foram obtidos pelo método $\mathrm{LMS}^{34}$, implementado no software LMSchartmaker Pro versão 2.5435. Para normalizar a distribuiçáo dos valores em cada uma das variáveis, o método LMS recorre à transformação Box-Cox, específica para cada idade, os valores L, M e S são Cubic Splines em cada intervalo etário. Este método consiste em três curvas suavizadas e específicas de cada idade, designadas de curva L (transformação BoxCox), curva M (mediana) e curva $S$ (coeficiente de variaçáo) com base na seguinte equação,

$$
\mathrm{C}_{100 \alpha}(\mathrm{t})=\mathrm{M}(\mathrm{t})\left[1+\mathrm{L}(\mathrm{t}) \mathrm{S}(\mathrm{t}) \mathrm{Z}_{\alpha}\right]^{1 / \mathrm{L}(\mathrm{t})}
$$

em que $Z \alpha$ é o desvio normal equivalente para a amostra total, $\alpha$ e C100 $\alpha(t)$ o percentil correspondente. A complexidade da suavização de cada curva foi medida pelos graus de liberdade equivalentes para $\mathrm{L}(\mathrm{t}), \mathrm{M}(\mathrm{t})$ e $\mathrm{S}(\mathrm{t})$. Foram utilizados testes $\mathrm{Q}^{35,36}$ para adequaçáo do ajustamento, bem como representaçóes de Worm plots ${ }^{34,37}$.
A TABELA 3 apresenta os valores percentílicos (P3, P10, P50; P75; P90; P97) de meninos e meninas, por idade e subteste do BOT-2. Na avaliação da precisão motora fina, o valor mediano (P50) de meninos e meninas aos dez anos é muito próximo, $\sim 34,04$ pontos. Igualmente no subteste de integração motora fina, as meninas possuem um valor mediano (P50) apenas 0,9 pontos superior em comparação aos meninos. $\mathrm{Na}$ avaliação da destreza manual, os valores do P50 dos meninos aos seis anos é de 17,84 pontos, enquanto que das meninas é de 18,85 pontos. O aumento é constante em todas as idades; aos 10 anos, as meninas apresentam um valor mediano de 28,69 pontos, enquanto os meninos de 24,75 pontos. No subteste de coordenação bilateral, verifica-se um aumento progressivo ao longo das idades para ambos os sexos, cujo valor mediano (P50) aos 6 anos para meninas e meninos é de 18,08 pontos e 16,94 pontos, respectivamente, chegando a 22,53 pontos e 22,76 pontos, respectivamente. $\mathrm{Na}$ avaliação do equilíbrio, há 
uma pequena variação dos valores do P50, entre 0,9 e 2,99 pontos, ao longo das idades em ambos os sexos. Todavia, os valores mais elevados são observados para as meninas ao longo de todas as idades, com exceção do P97, no qual meninos apresentam escores superiores a partir dos sete anos.

TABELA 2 - Média, desvio padrão e amplitude para os oito subtestes do BOT-2, segundo sexo e idade.

\begin{tabular}{|c|c|c|c|c|c|c|}
\hline \multirow{3}{*}{$\begin{array}{l}\text { Variáveis } \\
\text { (pontos) }\end{array}$} & \multirow{3}{*}{ Sexo } & \multicolumn{5}{|c|}{ Idades } \\
\hline & & 6 & 7 & 8 & 9 & 10 \\
\hline & & $\begin{array}{l}\text { média } \pm \mathrm{DP} \\
\text { min - max }\end{array}$ & $\begin{array}{c}\text { média } \pm \text { DP } \\
\text { min - max }\end{array}$ & $\begin{array}{l}\text { média } \pm \text { DP } \\
\text { min - max }\end{array}$ & $\begin{array}{l}\text { média } \pm \mathrm{DP} \\
\text { min - max }\end{array}$ & $\begin{array}{l}\text { média } \pm \text { DP } \\
\text { min - max }\end{array}$ \\
\hline \multirow{2}{*}{$\begin{array}{l}\text { Precisão } \\
\text { Motora Fina }\end{array}$} & Meninos & $\begin{array}{c}23,68 \pm 6,03 \\
10-35\end{array}$ & $\begin{array}{c}27,95 \pm 5,65 \\
11-36\end{array}$ & $\begin{array}{c}30,94 \pm 4,81 \\
18-40\end{array}$ & $\begin{array}{c}32,78 \pm 4,02 \\
22-39\end{array}$ & $\begin{array}{c}33,55 \pm 3,70 \\
20-40\end{array}$ \\
\hline & Meninas & $\begin{array}{c}26,94 \pm 5,71 \\
15-40\end{array}$ & $\begin{array}{c}29,58 \pm 4,38 \\
19-39\end{array}$ & $\begin{array}{c}32,66 \pm 3,77 \\
20-39\end{array}$ & $\begin{array}{c}33,61 \pm 3,51 \\
22-40\end{array}$ & $\begin{array}{c}34,97 \pm 3,04 \\
26-41\end{array}$ \\
\hline \multirow{2}{*}{$\begin{array}{l}\text { Integração } \\
\text { Motora Fina }\end{array}$} & Meninos & $\begin{array}{c}21,86 \pm 5,71 \\
10-35\end{array}$ & $\begin{array}{c}25,78 \pm 6,08 \\
7-36\end{array}$ & $\begin{array}{c}28,21 \pm 4,56 \\
15-39\end{array}$ & $\begin{array}{c}29,38 \pm 4,06 \\
17-39\end{array}$ & $\begin{array}{c}30,09 \pm 4,54 \\
15-38\end{array}$ \\
\hline & Meninas & $\begin{array}{c}23,90 \pm 5,67 \\
12-35\end{array}$ & $\begin{array}{c}26,68 \pm 5,11 \\
10-38\end{array}$ & $\begin{array}{c}29,26 \pm 4,19 \\
18-37\end{array}$ & $\begin{array}{c}30,18 \pm 3,70 \\
19-38\end{array}$ & $\begin{array}{c}31,56 \pm 4,11 \\
16-39\end{array}$ \\
\hline \multirow{2}{*}{$\begin{array}{l}\text { Destreza } \\
\text { Manual }\end{array}$} & Meninos & $\begin{array}{c}18,89 \pm 3,37 \\
12-26\end{array}$ & $\begin{array}{l}21,34 \pm 4,04 \\
\quad 8-31\end{array}$ & $\begin{array}{c}23,90 \pm 3,75 \\
15-34\end{array}$ & $\begin{array}{c}26,65 \pm 3,84 \\
14-35\end{array}$ & $\begin{array}{c}26,67 \pm 4,46 \\
11-36\end{array}$ \\
\hline & Meninas & $\begin{array}{c}19,88 \pm 3,46 \\
12-27\end{array}$ & $\begin{array}{c}22,86 \pm 3,75 \\
15-32\end{array}$ & $\begin{array}{c}25,98 \pm 3,47 \\
16-34\end{array}$ & $\begin{array}{c}27,73 \pm 4,03 \\
16-39\end{array}$ & $\begin{array}{c}29,29 \pm 4,13 \\
17-41\end{array}$ \\
\hline $\begin{array}{l}\text { Coordenação } \\
\text { Motora dos }\end{array}$ & Meninos & $\begin{array}{c}26,24 \pm 7,34 \\
10-37\end{array}$ & $\begin{array}{c}28,74 \pm 6,70 \\
9-39\end{array}$ & $\begin{array}{c}32,91 \pm 5,39 \\
15-39\end{array}$ & $\begin{array}{c}34,78 \pm 4,21 \\
12-39\end{array}$ & $\begin{array}{c}35,87 \pm 3,14 \\
24-39\end{array}$ \\
\hline $\begin{array}{l}\text { Membros } \\
\text { Superiores }\end{array}$ & Meninas & $\begin{array}{c}20,42 \pm 7,49 \\
4-38\end{array}$ & $\begin{array}{c}26,92 \pm 6,63 \\
6-38\end{array}$ & $\begin{array}{c}29,74 \pm 5,64 \\
10-39\end{array}$ & $\begin{array}{c}31,96 \pm 5,44 \\
3-39\end{array}$ & $\begin{array}{c}34,03 \pm 3,39 \\
26-39\end{array}$ \\
\hline \multirow{2}{*}{$\begin{array}{l}\text { Coordenação } \\
\text { Bilateral }\end{array}$} & Meninos & $\begin{array}{c}16,60 \pm 4,11 \\
6-23\end{array}$ & $\begin{array}{c}19,00 \pm 3,42 \\
8-24\end{array}$ & $\begin{array}{l}21,30 \pm 2,76 \\
8-24\end{array}$ & $\begin{array}{c}21,96 \pm 2,52 \\
10-24\end{array}$ & $\begin{array}{c}21,77 \pm 2,48 \\
15-24\end{array}$ \\
\hline & Meninas & $\begin{array}{c}18,08 \pm 3,65 \\
8-24\end{array}$ & $\begin{array}{c}20,39 \pm 3,20 \\
\quad 7-24\end{array}$ & $\begin{array}{c}21,10 \pm 3,15 \\
7-24\end{array}$ & $\begin{array}{c}21,81 \pm 2,20 \\
13-24\end{array}$ & $\begin{array}{c}22,33 \pm 1,90 \\
16-24\end{array}$ \\
\hline \multirow{2}{*}{ Equilíbrio } & Meninos & $\begin{array}{c}30,22 \pm 3,30 \\
22-35\end{array}$ & $\begin{array}{c}31,64 \pm 3,36 \\
20-37\end{array}$ & $\begin{array}{c}32,52 \pm 2,88 \\
23-37\end{array}$ & $\begin{array}{c}33,32 \pm 2,67 \\
26-37\end{array}$ & $\begin{array}{c}33,22 \pm 2,67 \\
25-37\end{array}$ \\
\hline & Meninas & $\begin{array}{c}30,94 \pm 3,10 \\
22-37\end{array}$ & $\begin{array}{c}32,25 \pm 2,55 \\
23-37\end{array}$ & $\begin{array}{c}33,21 \pm 2,57 \\
22-37\end{array}$ & $\begin{array}{c}33,44 \pm 2,71 \\
24-37\end{array}$ & $\begin{array}{c}33,56 \pm 2,48 \\
27-37\end{array}$ \\
\hline \multirow{2}{*}{$\begin{array}{l}\text { Velocidade e } \\
\text { Agilidade }\end{array}$} & Meninos & $\begin{array}{c}30,40 \pm 4,01 \\
23-39\end{array}$ & $\begin{array}{c}31,10 \pm 4,91 \\
16-42\end{array}$ & $\begin{array}{c}33,84 \pm 4,30 \\
20-44\end{array}$ & $\begin{array}{c}35,29 \pm 4,39 \\
23-48\end{array}$ & $\begin{array}{c}35,24 \pm 4,70 \\
16-44\end{array}$ \\
\hline & Meninas & $\begin{array}{c}29,20 \pm 4,60 \\
16-39\end{array}$ & $\begin{array}{c}30,91 \pm 4,02 \\
15-39\end{array}$ & $\begin{array}{c}32,78 \pm 3,96 \\
19-40\end{array}$ & $\begin{array}{c}34,34 \pm 4,11 \\
22-48\end{array}$ & $\begin{array}{c}34,89 \pm 3,51 \\
27-45\end{array}$ \\
\hline \multirow{2}{*}{$\begin{array}{l}\text { Força } \\
\text { Muscular }\end{array}$} & Meninos & $\begin{array}{c}16,42 \pm 5,27 \\
3-27\end{array}$ & $\begin{array}{c}18,73 \pm 5,26 \\
4-29\end{array}$ & $\begin{array}{c}20,65 \pm 4,90 \\
8-33\end{array}$ & $\begin{array}{c}22,35 \pm 5,21 \\
8-33\end{array}$ & $\begin{array}{c}22,65 \pm 5,57 \\
\quad 8-32\end{array}$ \\
\hline & Meninas & $\begin{array}{c}15,16 \pm 4,79 \\
4-27\end{array}$ & $\begin{array}{c}17,02 \pm 4,87 \\
5-27\end{array}$ & $\begin{array}{c}18,92 \pm 4,85 \\
8-29\end{array}$ & $\begin{array}{c}19,64 \pm 4,85 \\
8-29\end{array}$ & $\begin{array}{c}20,64 \pm 5,04 \\
8-33\end{array}$ \\
\hline
\end{tabular}

$\mathrm{DP}=$ desvio padrão; $\min =$ mínimo; max = máximo. 
Ferreira L, et al.

FIGURA 1 - Curvas percentílicas dos oitos subtestes do BOT-2 para meninos e meninas.
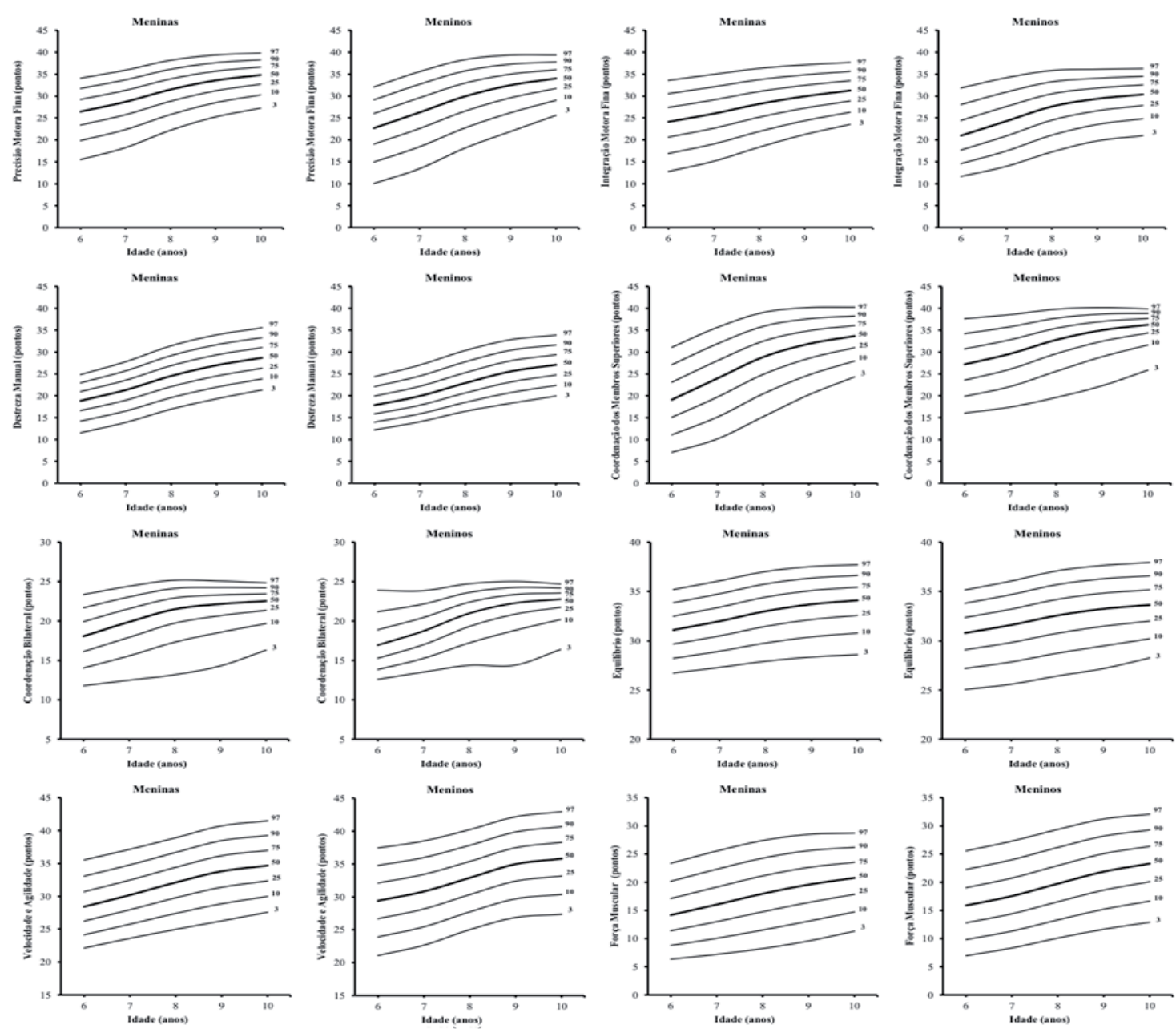
TABELA 3 - Valores numéricos dos percentis $\left(\mathrm{P}_{3}, \mathrm{P}_{10}, \mathrm{P}_{25}, \mathrm{P}_{50}, \mathrm{P}_{75}, \mathrm{P}_{90}, \mathrm{P}_{97}\right)$ dos subtestes do BOT-2 por sexo eidade.

\begin{tabular}{|c|c|c|c|c|c|c|c|c|c|c|c|c|c|c|}
\hline \multirow[t]{3}{*}{ Idade } & \multicolumn{7}{|c|}{ Meninos } & \multicolumn{7}{|c|}{ Meninas } \\
\hline & \multicolumn{14}{|c|}{ Precisáo Motora Fina (pontos) } \\
\hline & P3 & P10 & P25 & P50 & P75 & P90 & P97 & P3 & P10 & P25 & P50 & P75 & P90 & P97 \\
\hline 6 & 10,07 & 14,95 & 19,05 & 22,70 & 26,05 & 29,17 & 32,10 & 15,50 & 19,84 & 23,39 & 26,48 & 29,23 & 31,76 & 34,09 \\
\hline 7 & 13,43 & 18,43 & 22,60 & 26,28 & 29,62 & 32,71 & 35,60 & 18,25 & 22,37 & 25,76 & 28,70 & 31,33 & 33,73 & 35,94 \\
\hline 8 & 18,13 & 22,74 & 26,58 & 29,94 & 32,97 & 35,75 & 38,34 & 22,21 & 25,79 & 28,83 & 31,50 & 33,92 & 36,13 & 38,18 \\
\hline 9 & 21,92 & 26,21 & 29,61 & 32,49 & 35,02 & 37,29 & 39,36 & 25,24 & 28,49 & 31,21 & 33,58 & 35,69 & 37,62 & 39,38 \\
\hline 10 & 25,61 & 29,05 & 31,77 & 34,04 & 36,03 & 37,80 & 39,40 & 27,27 & 30,28 & 32,73 & 34,83 & 36,67 & 38,33 & 39,83 \\
\hline
\end{tabular}

\section{Integraçáo Motora Fina (pontos)}

$\begin{array}{lllllllllllllllll} & \text { P3 } & \text { P10 } & \text { P25 } & \text { P50 } & \text { P75 } & \text { P90 } & \text { P97 } & \text { P3 } & \text { P10 } & \text { P25 } & \text { P50 } & \text { P75 } & \text { P90 } & \text { P97 } \\ 6 & 11,67 & 14,58 & 17,68 & 20,98 & 24,45 & 28,09 & 31,88 & 12,79 & 16,89 & 20,63 & 24,11 & 27,41 & 30,56 & 33,59 \\ 7 & 13,97 & 17,48 & 20,92 & 24,30 & 27,64 & 30,94 & 34,21 & 15,13 & 19,08 & 22,67 & 26,00 & 29,14 & 32,13 & 34,98 \\ 8 & 17,25 & 21,08 & 24,50 & 27,63 & 30,53 & 33,27 & 35,86 & 18,34 & 21,92 & 25,18 & 28,21 & 31,06 & 33,76 & 36,34 \\ 9 & 19,76 & 23,52 & 26,65 & 29,39 & 31,84 & 34,08 & 36,15 & 21,22 & 24,37 & 27,26 & 29,94 & 32,47 & 34,86 & 37,14 \\ 10 & 20,95 & 24,84 & 27,86 & 30,38 & 32,57 & 34,53 & 36,30 & 23,54 & 26,32 & 28,89 & 31,28 & 33,53 & 35,66 & 37,69\end{array}$

Destreza Manual (pontos)

$\begin{array}{ccccccccccccccccc} & \text { P3 } & \text { P10 } & \text { P25 } & \text { P50 } & \text { P75 } & \text { P90 } & \text { P97 } & \text { P3 } & \text { P10 } & \text { P25 } & \text { P50 } & \text { P75 } & \text { P90 } & \text { P97 } \\ 6 & 12,20 & 13,99 & 15,87 & 17,84 & 19,92 & 22,09 & 24,35 & 11,53 & 14,20 & 16,61 & 18,85 & 20,95 & 22,94 & 24,84 \\ 7 & 14,06 & 15,89 & 17,84 & 19,93 & 22,16 & 24,53 & 27,03 & 13,92 & 16,54 & 18,99 & 21,33 & 23,56 & 25,72 & 27,80 \\ 8 & 16,44 & 18,48 & 20,63 & 22,89 & 25,27 & 27,75 & 30,35 & 16,95 & 19,57 & 22,09 & 24,52 & 26,89 & 29,19 & 31,44 \\ 9 & 18,28 & 20,71 & 23,13 & 25,56 & 27,98 & 30,41 & 32,83 & 19,30 & 21,95 & 24,51 & 26,97 & 29,35 & 31,67 & 33,93 \\ 10 & 19,93 & 22,36 & 24,75 & 27,08 & 29,38 & 31,64 & 33,87 & 21,33 & 23,85 & 26,30 & 28,69 & 31,03 & 33,31 & 35,56\end{array}$

Coordenaçáo dos Membros Superiores (pontos)

$\begin{array}{cccccccccccccccc} & \mathbf{P 3} & \mathbf{P 1 0} & \mathbf{P 2 5} & \mathbf{P 5 0} & \mathbf{P 7 5} & \mathbf{P 9 0} & \mathbf{P 9 7} & \mathbf{P 3} & \mathbf{P 1 0} & \mathbf{P 2 5} & \mathbf{P 5 0} & \text { P75 } & \text { P90 } & \text { P97 } \\ \mathbf{6} & 16,05 & 19,85 & 23,56 & 27,17 & 30,72 & 34,21 & 37,65 & 7,08 & 11,09 & 15,09 & 19,10 & 23,10 & 27,10 & 31,10 \\ 7 & 17,43 & 22,12 & 26,10 & 29,63 & 32,84 & 35,80 & 38,58 & 10,15 & 15,16 & 19,72 & 23,98 & 28,03 & 31,91 & 35,66 \\ 8 & 19,61 & 25,59 & 29,63 & 32,79 & 35,45 & 37,75 & 39,81 & 15,23 & 20,44 & 24,89 & 28,85 & 32,49 & 35,87 & 39,06 \\ 9 & 22,28 & 28,92 & 32,47 & 35,01 & 37,02 & 38,70 & 40,16 & 20,23 & 24,76 & 28,56 & 31,88 & 34,88 & 37,62 & 40,17 \\ 10 & 25,85 & 31,63 & 34,37 & 36,24 & 37,69 & 38,88 & 39,89 & 24,31 & 27,95 & 31,01 & 33,68 & 36,07 & 38,25 & 40,27\end{array}$

Coordenaçáo Bilateral (pontos)

\begin{tabular}{cccccccccccccccc} 
& P3 & P10 & P25 & P50 & P75 & P90 & P97 & P3 & P10 & P25 & P50 & P75 & P90 & P97 \\
6 & 12,60 & 13,84 & 15,28 & 16,94 & 18,88 & 21,17 & 23,88 & 11,79 & 14,05 & 16,13 & 18,08 & 19,92 & 21,67 & 23,36 \\
7 & 13,52 & 15,26 & 17,00 & 18,72 & 20,44 & 22,14 & 23,84 & 12,49 & 15,59 & 17,94 & 19,88 & 21,56 & 23,05 & 24,41 \\
8 & 14,37 & 17,22 & 19,29 & 20,95 & 22,37 & 23,61 & 24,72 & 13,19 & 17,32 & 19,72 & 21,49 & 22,91 & 24,12 & 25,18 \\
9 & 14,40 & 18,80 & 20,85 & 22,25 & 23,34 & 24,24 & 25,01 & 14,31 & 18,57 & 20,68 & 22,15 & 23,29 & 24,25 & 25,07 \\
10 & 16,38 & 20,18 & 21,73 & 22,76 & 23,54 & 24,17 & 24,70 & 16,31 & 19,66 & 21,35 & 22,53 & 23,44 & 24,19 & 24,83 \\
\hline
\end{tabular}

Continua

Rev Bras Educ Fís Esporte, (São Paulo) 2020 Out-Dez;34(4):639-52 • 645 


\begin{tabular}{|c|c|c|c|c|c|c|c|c|c|c|c|c|c|c|}
\hline \multirow{3}{*}{ Idade } & \multicolumn{7}{|c|}{ Meninos } & \multicolumn{7}{|c|}{ Meninas } \\
\hline & \multicolumn{14}{|c|}{ Equilíbrio (pontos) } \\
\hline & P3 & P10 & P25 & P50 & P75 & P90 & P97 & P3 & P10 & P25 & P50 & P75 & P90 & P97 \\
\hline 6 & 25,04 & 27,19 & 29,08 & 30,79 & 32,35 & 33,80 & 35,14 & 26,73 & 28,22 & 29,67 & 31,09 & 32,49 & 33,85 & 35,19 \\
\hline 7 & 25,59 & 27,85 & 29,82 & 31,59 & 33,20 & 34,68 & 36,05 & 27,29 & 28,93 & 30,49 & 31,97 & 33,39 & 34,76 & 36,07 \\
\hline 8 & 26,42 & 28,74 & 30,75 & 32,56 & 34,19 & 35,69 & 37,09 & 27,92 & 29,77 & 31,44 & 32,99 & 34,42 & 35,75 & 37,01 \\
\hline 9 & 27,18 & 29,48 & 31,46 & 33,23 & 34,82 & 36,29 & 37,64 & 28,35 & 30,39 & 32,14 & 33,69 & 35,08 & 36,35 & 37,52 \\
\hline \multirow[t]{3}{*}{10} & 28,25 & 30,20 & 31,98 & 33,62 & 35,15 & 36,58 & 37,94 & 28,59 & 30,78 & 32,57 & 34,09 & 35,43 & 36,62 & 37,71 \\
\hline & \multicolumn{14}{|c|}{ Velocidade e Agilidade (pontos) } \\
\hline & P3 & P10 & P25 & P50 & P75 & P90 & P97 & P3 & P10 & P25 & P50 & P75 & P90 & P97 \\
\hline 6 & 21,07 & 23,90 & 26,68 & 29,42 & 32,12 & 34,79 & 37,44 & 22,12 & 24,13 & 26,23 & 28,42 & 30,71 & 33,08 & 35,54 \\
\hline 7 & 22,64 & 25,44 & 28,17 & 30,83 & 33,44 & 36,00 & 38,51 & 23,60 & 25,76 & 27,96 & 30,20 & 32,48 & 34,80 & 37,15 \\
\hline 8 & 25,01 & 27,72 & 30,35 & 32,91 & 35,40 & 37,84 & 40,24 & 24,97 & 27,40 & 29,78 & 32,12 & 34,42 & 36,68 & 38,91 \\
\hline 9 & 26,86 & 29,70 & 32,40 & 34,99 & 37,47 & 39,87 & 42,19 & 26,26 & 28,87 & 31,38 & 33,81 & 36,18 & 38,48 & 40,72 \\
\hline \multirow[t]{3}{*}{10} & 27,34 & 30,37 & 33,18 & 35,81 & 38,31 & 40,68 & 42,95 & 27,58 & 29,99 & 32,36 & 34,69 & 36,98 & 39,25 & 41,49 \\
\hline & \multicolumn{14}{|c|}{ Força Muscular (pontos) } \\
\hline & P3 & P10 & P25 & P50 & P75 & P90 & P97 & P3 & P10 & P25 & P50 & P75 & P90 & P97 \\
\hline 6 & 6,95 & 9,81 & 12,80 & 15,88 & 19,04 & 22,27 & 25,57 & 6,33 & 8,77 & 11,39 & 14,18 & 17,11 & 20,18 & 23,38 \\
\hline 7 & 8,33 & 11,32 & 14,40 & 17,55 & 20,75 & 24,01 & 27,30 & 7,19 & 10,05 & 13,00 & 16,04 & 19,15 & 22,31 & 25,54 \\
\hline 8 & 10,08 & 13,31 & 16,54 & 19,76 & 22,97 & 26,17 & 29,37 & 8,22 & 11,51 & 14,75 & 17,96 & 21,13 & 24,27 & 27,40 \\
\hline 9 & 11,65 & 15,20 & 18,60 & 21,88 & 25,07 & 28,19 & 31,23 & 9,57 & 13,09 & 16,41 & 19,58 & 22,63 & 25,60 & 28,49 \\
\hline 10 & 12,90 & 16,66 & 20,10 & 23,31 & 26,36 & 29,27 & 32,07 & 11,30 & 14,73 & 17,86 & 20,78 & 23,55 & 26,19 & 28,73 \\
\hline
\end{tabular}

Para o subteste de coordenação dos membros superiores, os meninos têm melhores desempenhos em comparação às meninas. Essa diferença foi observada em todas as idades. No subteste velocidade e agilidade, o valor mediano (P50) dos meninos de seis anos é 29,42 pontos e aos 10 anos é de 35,81 pontos, enquanto que nas meninas é de 28,42 pontos aos seis anos e de 34,69 pontos aos 10 anos. Na força muscular, observa-se um comportamento bem distinto entre as curvas percentílicas de meninos e meninas; os meninos apresentam melhores resultados em todas as idades.

A comparação dos valores médios das crianças maringaenses com a referência baseada nos escores das crianças norte-americanas, proposto no manual do BOT-217 para os oitos subtestes, é apresentada na FIGURA 2.

Nos subtestes de precisão motora fina, integração motora fina, destreza manual, e força muscular, as crianças norte-americanas apresentam desempenho superior. Os resultados superiores das crianças americanas comparativamente às brasileiras variam entre 0,33 e 6,69 pontos; por exemplo, a maior diferença é de 6,69 pontos para os meninos norteamericanos no subteste de integração motora fina aos 10 anos de idade. Todavia, na avaliação da destreza manual, as curvas se sobrepóem; as meninas aos oito anos e os meninos aos seis e nove anos apresentam resultados médios similares. 
FIGURA 2 - Comparação dos valores médios dos oitos subtestes do BOT-2 deste estudo com os valores apresentado no manual original do teste $(n=560)$.
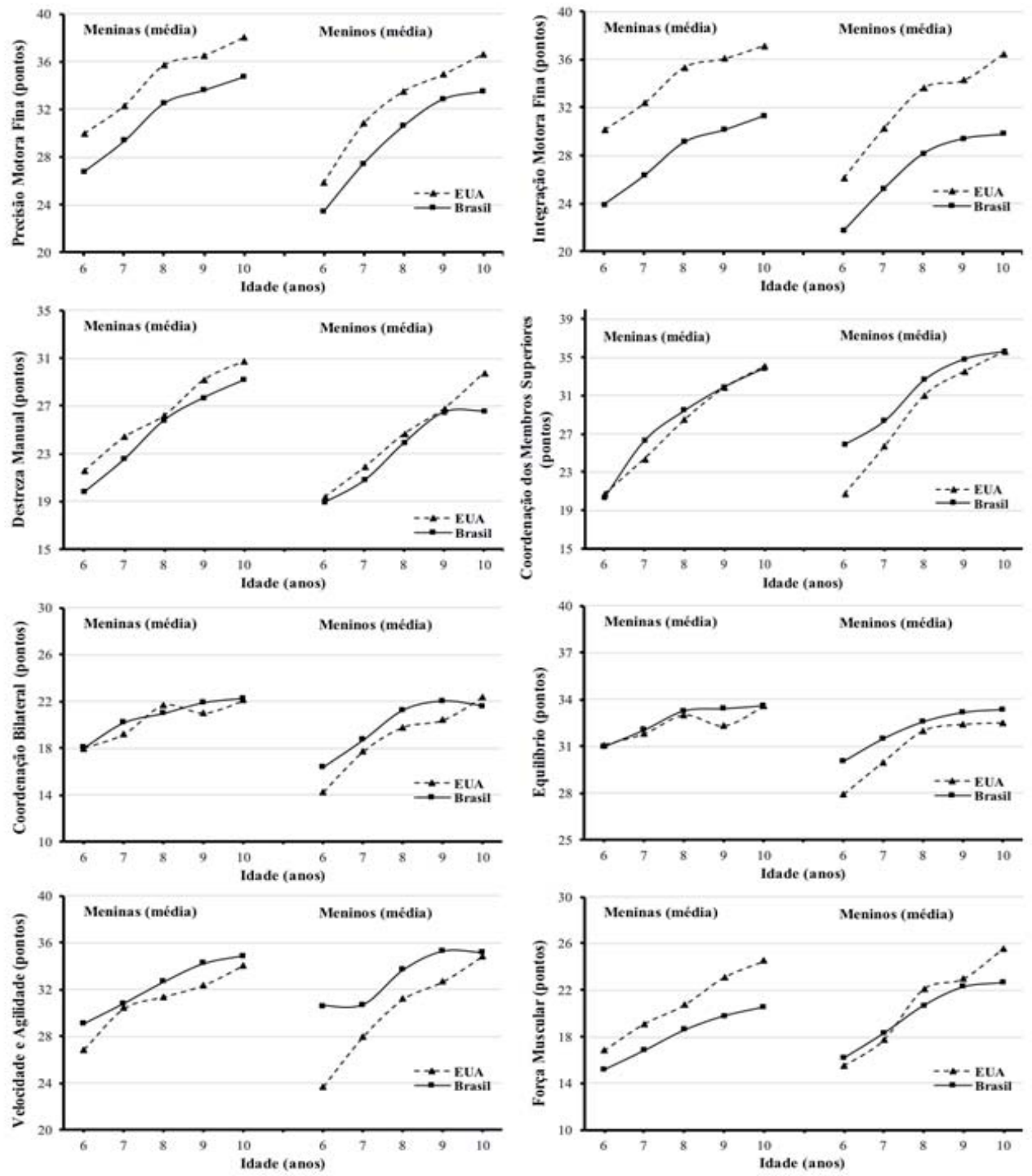

Por outro lado, as crianças maringaenses mostram melhores resultados nos subtestes de coordenação bilateral, equilíbrio, coordenação dos membros superiores, e velocidade e agilidade, com amplitude de diferença variando entre 0,03 e 6,90 pontos; por exemplo, a maior diferença encontrada foi de 6,90 pontos na velocidade e agilidade para os meninos aos seis anos de idade. Com exceção dos resultados da avaliação da coordenação bilateral, aos oito anos para as meninas e aos 10 anos para os meninos, onde há uma inversão da curva e, assim, melhor desempenho das crianças norte-americanas.

Nos subtestes de coordenação dos membros superiores, as curvas se sobrepóem em algumas idades, nomeadamente aos seis, nove e 10 anos para as meninas e aos 10 anos para os meninos, apresentando valores médios similares em ambos os grupos. O mesmo acontece para o subteste de Equilíbrio nas meninas aos seis anos, conforme observado na FIGURA 2.

Estima-se que os valores abaixo do percentil 10 correspondam a uma baixa proficiência motora, valores entre o percentil 10 e 90 sejam considerados adequados para cada idade e sexo, e que os valores acima do percentil 90 sejam considerados superiores (TABELA 3). 


\section{Discussão}

Este estudo teve como objetivos construir valores de referência percentílicas para os oito subtestes do BOT-2, e comparar a proficiência motora das crianças maringaenses com o desempenho das crianças norte-americanas do estudo de referência ${ }^{17}$.

A trajetória das curvas percentílicas sugerem o aumento dos níveis de proficiência motora com o avanço da idade, em ambos os sexos, corroborando estudos prévios $^{26-29}$. Logan et al. ${ }^{38}$ destacam que o desempenho das habilidades motoras tende a aumentar ao longo da idade devido à participação mais efetiva das crianças em programas de educação física, com instruçôes sobre técnica de habilidades motoras grossas.

É importante ressaltar que no presente estudo o aumento entre os seis e os oitos anos; após essa idade, as curvas mostraram incrementos constantes na pontuação dos subtestes, ou um plateau. CHAVEs et al. ${ }^{26}$, ao analisarem as pseudo-curvas de velocidade para os quatro testes da bateria KTK, sugerem que pode existir redução da velocidade dos incrementos anuais da proficiência motora entre os seis e os 10 anos de idade, cujas mudanças nas pontuaçóes anuais são específicas de cada teste e podem estar associadas à existência de janelas de oportunidades de aprendizagem motora. Contudo, não é de nosso conhecimento estudos que tenham construído cartas de velocidade da proficiência motora para que seja possível uma interpretação mais profunda.

A forte variabilidade interindividual é expressa na amplitude dos valores situados entre o P3 e o P97. Por exemplo, para a força muscular, a amplitude dos valores percentílicos aos seis anos varia entre 6,95 pontos (P3) e 25,57 pontos (P97) para os meninos, e 6,33 pontos (P3) e 23,38 pontos (P97) para as meninas. Essa variação ilustra que na mesma idade existem crianças com baixa proficiência motora e outras que apresentam valores próximos ou superiores ao percentil 50 (P50), ou seja, expressivas diferenças quanto aos níveis de proficiência motora entre crianças da mesma idade. Essas diferenças encontradas podem ocorrer devido as características biológicas individuais ${ }^{39}$, ao tipo de experiência adquirida que afetam as mudanças no desenvolvimento que ocorrem durante um período específico, bem como influenciadas por restriçôes culturais, familiares e sociais ${ }^{40}$.

Considerando as diferenças no desempenho dos subtestes do BOT2 entre os sexos, as meninas apresentaram melhor desempenho na precisão motora fina, integração motora fina, destreza manual e equilíbrio. De forma similar o estudo de Miyabayashi e Pimentel ${ }^{41}$ com crianças brasileiras as meninas apresentaram valores superiores no equilíbrio, resultados semelhantes para meninas gregas $^{42}$ e turcas ${ }^{43}$, com um melhor desempenho em atividades que envolvem destreza manual e equilíbrio, e resultado diferente do estudo de YengDe et al. ${ }^{44}$, no qual o desempenho dos meninos indianos foi melhor do que das meninas indianas no equilíbrio.

Em contrapartida, os meninos apresentaram melhor desempenho na coordenação dos membros superiores, velocidade e agilidade e força muscular. Resultados similares foram reportados por Miyabayashi e Pimentel ${ }^{41}$ e MülazimoĞLuBALLI $^{43}$ com os meninos brasileiros e gregos, respectivamente, apresentando valores superiores na velocidade e agilidade e força muscular. No estudo de CHur et al. ${ }^{45}$ melhores valores foram encontrados na coordenaçáo de membros superiores dos meninos de Hong Kong e Estados Unidos. Já Morley et al. ${ }^{46}$ encontraram melhores desempenhos para as meninas na Inglaterra na velocidade e agilidade, ao analisar crianças de 6 e 7 anos.

As diferenças encontradas podem ser justificadas pelos diferentes níveis de atividade física realizados por meninos e meninas ${ }^{47}$. Estudos ${ }^{48,49}$ têm reportado que meninos apresentaram níveis de atividade física e participaçáo esportiva significativamente maiores do que as meninas. Culturalmente, os meninos são mais encorajados a praticar esportes ${ }^{50}$ em comparação as meninas que são pouco incentivadas ${ }^{51}$. Já, as meninas são orientadas para práticas de atividades manuais, com bonecas e brinquedos educativo ${ }^{52}$ e com maior atribuição trabalho doméstico e familiar ${ }^{51}$. Aspectos motivacionais, sobretudo nas meninas, que com o aumento da idade tendem a adotar comportamentos menos ativos podem contribuir para explicação dos resultados encontrados ${ }^{53}$.

Ao comparar os resultados deste estudo com a pontuaçấo de referência descrita no manual do BOT-2, verifica-se que as crianças maringaenses mostram desempenho superior em quatro subtestes do BOT-2, nomeadamente coordenação bilateral, velocidade e agilidade, equilíbrio e coordenaçáo dos membros superiores. Resultados podem ser explicados devido a fatores socioculturais, SINGER et al. ${ }^{54}$ ao questionar sobre a prática de jogos infantis para famílias brasileiras e americanas, destacou que 
as mães de crianças brasileiras (69\%) têm maior preocupação com participação das crianças em atividades ao ar livre que as mães de crianças norteamericanas (12\%).

As crianças americanas, por sua vez, apresentaram escores mais elevados em tarefas que exigem a coordenação motora fina (precisão motora fina, integração motora fina, destreza manual) e força muscular. A amplitude dessas diferenças situa-se entre 3,21 pontos (meninas aos seis anos) e 3,25 pontos (meninas aos 10 anos) na precisão motora fina por exemplo. Corroborando os resultados encontrados, recentemente, o estudo de VAlEntini et $\mathrm{al} .{ }^{47}$ demonstrou que as crianças norte-americanas apresentaram nível mais elevado de proficiência motora total e melhor desempenho nos subtestes de destreza manual e atividade com bola em comparação com as crianças maringaenses, quando avaliadas utilizando o MABC.

Para Singer et al. ${ }^{54}$, crianças americanas já não se envolvem tanto em atividades ativas, pois trocaram por atividades de tela pequena, como jogos de vídeo e computadores, o que pode estar auxiliando no desenvolvendo da coordenação motora fina. Explicaçóes plausíveis para as diferenças encontradas podem estar relacionadas às oportunidades restritas no Brasil para que as crianças desenvolvam habilidades motoras grossas e finas ${ }^{47,55}$.

Segundo Valentini, Clark e Whitall ${ }^{55}$, especificamente, no Brasil, os serviços profissionais públicos e as oportunidades para participar de programas de intervenção precoce são restritos. Além disso, não é obrigatório que as aulas de educação física sejam ministradas por professores formados em educação física ${ }^{47}$. Todavia, outros fatores também interferem nos desempenhos da proficiência motora, como as rotinas de vida diária, fatores socioculturais de cada população ${ }^{51}$ e a motivação intrínseca de cada crianç ${ }^{56}$. Um ou mais fatores combinados podem aumentar as chances de diferentes competências apresentadas por ambas as populaçóes.

Vários aspectos deste estudo merecem ser considerados ao avaliar as implicaçóes práticas dos resultados. Primeiramente, sua evidente aplicação como referência para descrição da proficiência motora de crianças, com valores normativos específicos por sexo e idade considerando populaçóes com características semelhantes a amostra do presente estudo, fato que pode contribuir significativamente para a adequação de programas de intervenção e avaliação da proficiência motora. Além disso, é indiscutível a contribuição em termos pedagógicos e clínicos, auxiliando profissionais da Educação Física e áreas afins. É importante salientar que a dimensão amostral, entre sexos e por intervalos de idade, permitiu que as estimativas dos percentis fossem precisas e robustas face ao procedimento de estimação implementado no método de Cole e GreEN ${ }^{34}$.

No que se refere às limitaçóes, o presente estudo permite estabelecer valores normativos para os oito subtestes para a população de Maringá (PR). A comparação com outras populaçóes deve considerar diferenças contextuais. No entanto, acredita-se que os benefícios deste estudo podem superar as limitações, sendo este o primeiro estudo no Brasil que estabeleceu cartas percentílicas para o BOT-2, com crianças em idade escolar (seis a 10 anos).

Em resumo, os valores de referência percentílicas construídos pelo presente estudo sugerem a forte variabilidade interindividual da proficiência motora em diferentes subtestes do BOT-2. Os resultados foram heterogêneos para meninos e meninas, e para crianças maringaenses em comparaçáo com as norte-americanas. Em geral, as curvas sugerem as especificidades para cada subteste e sexo, bem como o aumento nas pontuaçóes de cada teste ao longo das idades.

O uso dos valores normativos apresentados neste estudo pode auxiliar na interpretação da proficiência motora de crianças com idades entre os seis e os 10 anos, bem como na elaboração de intervençóes motoras mais efetivas. Para uma ampla caracterização nacional da proficiência motora, ainda se faz necessário a realização de novos estudos, envolvendo diferentes regióes, com uma amostra estratificada de todo o país. 


\begin{abstract}
Percentile charts for motor proficiency of children aged between six and 10 years.

This study had as main objectives (i) to present normative values for the eight subtests of the Bruininks-Oseretsky Motor Proficiency Test (BOT-2) and (ii) to contrast with the values suggested in the original test manual. The sample consisted of 931 children aged 6 to 10 years, of both sexes, from the city of Maringá-PR. The BOT-2 was used to assess motor proficiency. The construction of the percentile reference values was performed based on the LMS method, implemented by the LMSchartmaker Pro software version 2.54. The results show that girls scored higher on fine motor precision, fine motor integration, manual dexterity, and balance. The boys had higher scores on upper-limb coordination, running speed and agility, and strength. Brazilian children showed higher values than the North American children in the subtests of bilateral coordination, running speed and agility, balance, and upper-limb coordination. The results demonstrate the strong variability in terms of motor proficiency, as well as significant differences between boys and girls. The curves illustrate the increase in levels of motor proficiency across the ages, with different trajectories for each subtest. Thus, the use of the normative values presented in this study may help pedagogical action in schools and sport contexts, contributing to the planning of more effective motor interventions.
\end{abstract}

KEYWORDS: Motor proficiency; Bruininks-Oseretsky; Reference values.

\title{
Referências
}

1. Gabbard C. Lifelong motor development. San Franciso: Pearson; 2008.

2. Gallahue DL, Ozmun JC, Goodway JD. Compreendendo o desenvolvimento motor: bebês, crianças, adolescentes e adultos. Porto Alegre: AMGH; 2013.

3. Melnik BC, John SM, Schmitz G. Milk consumption during pregnancy increases birth weight, a risk factor for the development of diseases of civilization. J Transl Med. 2015;13:1-12. doi: 10.1186/s12967-014-0377-9.

4. Logan SW, Ross SM, Chee K, Stodden DF, Robinson LE. Fundamental motor skills: a systematic review of terminology. J Sports Sci. 2017;21:1-16. doi: 10.1080/02640414.2017.1340660.

5. Bart O, Jarus T, Erez Y, Rosenberg L. How do young children with DCD participate and enjoy daily activities? Res Dev Disabil 2011;32:1317-1322.

6. Mancini VO, Rigoli D, Cairney J, Roberts LD, Piek JP. The elaborated environmental stress hypothesis as a framework for understanding the association between motor skills and internalizing problems: a mini-review. Front Psychol. 2016;7:1-6.

7. Lingam R, Jongmans MJ, Ellis M, Hunt LP, Golding J, Emond A. Mental health difficulties in children with developmental coordination disorder. Pediatrics. 2012;129: e882-e891.

8. Hill EL, Brown D, Sorgardt K. A preliminary investigation of quality of life satisfaction reports in emerging adults with and without developmental coordination disorder. J Adult Dev. 2011;18:130-134.

9. Hardy LL, Reinten-Reynolds T, Espinel P, Zask A, Okely AD. Prevalence and correlates of low fundamental movement skill competency in children. Pediatrics. 2012;130:390-8.

10. Cattuzzo MT, Henrique RS, Réb AHN, Oliveira IS, Melo BM, Moura MS, et al. Motor competence and health related physical fitness in youth: a systematic review. J Sci Med Sport. 2016;19:123-129.

11. D'Hondt E, Deforche B, Bourdeaudhuij I, Lenoir M. Childhood obesity affects fine motor skill performance under different postural constraints. Neurosci Lett. 2008;440:72-75.

12. Clark JS. Models for Ecological Data: An Introduction. London: Paperback Lab Manual; 2007.

13. Kambas A, Venetsanou F, Giannakidou D, Fatouros IG, Avloniti A, Chatzinikolaou A, et al. The motorproficiencytest for children between 4 and 6 years of age (MOT 4-6): an investigation of its suitability in Greece. Res Dev Disabil. 2012;33:1626-1632.

14. Ulrich DA. The Test of Gross Motor Development - 3 (TGMD-3): Administration, scoring, and international norms. Spor Bilimleri Dergisi. 2013;24:27-33. 
15. Henderson SE, Sugden DA, Barnett AL. Movement assessment battery for children second edition. London: Psychological Corporation; 2007.

16. Kiphard EJ, Schilling VF. Körper-koordinations-test für kinder KTK: manual Von Fridhelm Schilling. Weinhein: Beltz Test; 1974.

17. Bruininks R, Bruininks B. Bruininks-Oseretsky test of motor proficiency, second edition. Minneapolis: NCS Pearson; 2005.

18. Cools W, Martelaer K, Samaey C, Andries C. Movement skill assessment of typically developing preschool children: A review of seven movement skill assessment tools. J Sports Sci Med. 2009;8:154-168.

19. Effgen S K. Meeting the Physical Therapy Needs of Children. Philadelphia: Davis Company; 2012.

20. McIntyre F, Parker H, Thornton A, Licari M, Piek J, Rigoli D, Hands B. Assessing motor proficiency in young adults: the Bruininks Oseretsky Test-2 Short Form and the McCarron Assessment of Neuromuscular Development. Hum Mov Sci. 53 (2017) 55-62

21. Kennedy J, Brown T, Stagnitti K. Top-down and bottom-up approaches to motor skill assessment of children: Are child-report and parent-report perceptions predictive of children's performance-based assessment results? Scand J Occup Ther. 2013;20:45-53.

22. Brown T, Rodger S, Brown A, Roever C. A profile of Canadian pediatric occupational therapy practice. Occup Ther Health Care. 2007;21:39-69.

23. Wuang Y, Lin Y, Su C. Rasch analysis of the Bruininks-Oseretsky test of motor proficiency-second edition in intellectual disabilities. Res Dev Disabil. 2009;30:1132-1144.

24. Bouchard C, Malina RM, Pérusse L. Genetics of fitness and physical performance. Champaign: Human Kinetics; 1997.

25. Chaves RN, Tani G, Souza MC, Santos D, Maia J. Variabilidade na coordenação motora: uma abordagem centrada no delineamento gemelar. Rev Bras Educ Fís Esporte. 2012;26:301-311.

26. Chaves RN, Tani G, Souza MC, Baxter-Jones A, Maia J. Desempenho coordenativo de crianças: construção de cartas percentílicas baseadas no método LMS de Cole e Green. Rev Bras Educ Fís Esporte. 2013;27:1-17.

27. Vidal SM, Bustamante A, Lopes VP, Seabra A, Silva RG, Maia JA. Construção de cartas centílicas da coordenação motora de crianças dos 6 aos 11 anos da Região Autónoma dos Açores, Portugal. Rev Port Cien Desp. 2008;9:24-35. 28. Vandorpe B, Vandendriessche J, Lefevre J, et al. The KorperkoordinationsTest fur Kinder: reference values and suitability for 6-12-year-old children in Flanders. Scand J Med Sci Sports, 2011;21:378-88.

29. Valdívia AB, Lara R, Espinoza CB, et al. Prontitud coordinativa: perfiles multivariados en función de la edad, sexo y estatus socio-económico. Rev Port Cien Desp. 2008;8:34-46.

30. Largo RH, Fischer JE, Rousson V. Neuromotor development from kindergarten age to adolescence: developmental course and variability. Swiss Med Wkly. 2003;133:193-97.

31. Silva S, Beunen G, Maia J. Valores normativos do desempenho motor de crianças e adolescentes: o estudo longitudinal-misto do Cariri. Rev Bras Educ Fís Esporte. 2011;25:111-25.

32. Milani S, Buckler JM, Kelnar CJ, et al. The use of local reference growth charts for clinical use or a universal standard: a balanced appraisal. J Endocrinol Invest. 2012;35:224-226.

33. Motta VT. Bioestatística. 2 ed. Caxias do Sul: Educs; 2006.

34. Cole TJ, Green PJ. Smoothing reference centile curves: the LMS method and penalized likelihood. Stat Med. 1992;11:1305-1319.

35. Pan H, Cole TJ. A comparison of goodness of fit tests for age-related reference ranges. Stat Med. 2004;23:17491765 .

36. Royston P, Wright EM. Goodness-of-fit statistics for age-specific reference intervals. Stat Med. 2000;19:29432962.

37. Buuren S, Fredriks M. Worm plot: a simple diagnostic device for modelling growth reference curves. Stat Med. 2001;20:1259-1277.

38. Logan SW, Robinson, LE, Wilson AE, Lucas, WA. Getting the fundamentals of movement: A meta-analysis of the effectiveness of motor skill interventions in children. Child Care Health Dev. 2011;38:305-315.

39. Guedes DP. Crescimento e desenvolvimento aplicado à Educaçáo Física e ao Esporte. Rev Bras Educ Fís Esporte. 2011;25:127-40.

40. Clark JE, Metcalfe J. The mountain of motor development: A metaphor. In: Clark JE, Humphrey J, organizers. 
Motor development: Research and reviews. Reston, VA: NASPE Publications; 2002, p. 163-90.

41. Miyabayashi LA, Pimentel GGA. Interaçôes sociais e proficiência motora em escolares do ensino fundamental. Rev Bras Educ Fís Esporte, 2011;25(4):649-62.

42. Venetsanou F, Kambas A. Motor Proficiency in Young Children: A Closer Look at Potential Gender Differences. Sage Open. 2016;6:1-10.

43. Mülazimoğlu-Balli Ö. Motor proficiency and body mass index of preschool children: in relation to socioeconomic status. J Educ Train Stud. 2016;4:237-43.

44. Yengde PN, Dhote S, Palekar T, Dighe A, Pande A. Assessment of balance using bot-2 in age group 5-15-year school going children. Asian Pac J Health Sci. 2017;4:152-160.

45. Chui MMY, Ng AMY, Fong AKH, Lin LSY, Ng MWF. Differences in the fine motor performance of children in Hong Kong and the United States on the Bruininks-Oseretsky Test of Motor Proficiency. HKJOT. 2007;17:1-9. 46. Morley D, Till K, Ogilvie P, Turner G. Influences of gender and socioeconomic status on the motor proficiency of children in the UK. Hum Mov Sci. 2015;44:150-156.

47. Valentini NC, Oliveira MA, Pangelinan MM, Whitall J, Clark JE. Can the MABC discriminate and predict motor impairment? a comparison of Brazilian and North American children. Int J Ther Rehabil. 2017;24:1-27.

48. Marques A, Ekelund U, Sardinha LB. Associations between organized sports participation and objectively measured physical activity, sedentary time and weight status in youth. J Sci Med Sport. 2016;19:154-57.

49. Hebert JJ, Møller NC, Andersen LB, Wedderkopp N. Organized sport participation is associated with higher levels of overall health-related physical activity in children (CHAMPS Study-DK). PLoS One. 2015;10: e0134621. doi:10.1371/journal. pone.0134621

50. Spessato BC, Gabbard C, Robinson L, Valentini NC. Body mass index, perceived and actual physical competence: the relationship among young children. Child Care Health Dev. 2013;39:845-50.

51. Valentini NC, Ramalho MH, Oliveira MA. Movement assessment battery for children-2: translation, reliability, and validity for Brazilian children. Res Dev Disabil. 2014;35:733-740.

52. Cherney ID, Lond K. Gender-linked differences in the toys, television shows, computer games, and outdoor activities of 5- to 13-year-old children. Sex Roles. 2006;54:717-726. doi:10.1007/s11199-006-9037-8.

53. Telama R, Yang X. Decline of physical activity from youth to young adulthood in Finland. Med Sci Sports Exerc. 2000;32:1617-22.

54. Singer DG, Singer JL, D’agostino H, Delong R. Children's Pastimes and Play in Sixteen Nations Is Free-Play Declining? Am J Play. 2009;1:283-12.

55. Valentini NC, Clark JE, Whitall J. Developmental co-ordination disorder in socially disadvantaged Brazilian children. Child Care Health Dev. 2014;41:970-979.

56. Bardid F, De Meester A, Tallir I, Cardon G, Lenoir M, Haerens L. Configurations of actual and perceived motor competence among children: Associations with motivation for sports and global self-worth. Hum Mov Sci. 2016;50:1-9. doi:10.1016/j.humov.2016.09.001.

ENDEREÇO

Luciana Ferreira

Av. Londrina, 934. Zona 8

87050-730 - Maringá - PR - Brasil E-mail: luferreira.ed@gmail.com
Submetido: $14 / 08 / 2017$

Revisado: 30/03/2018

Aceito: 07/ 05/ 2018 